\title{
Emergent contradictions: A synthesis of TRIZ and complex systems theory
}

\author{
Darrell Mann \\ 5A Yeo-Bank Business Park, Kenn Road, Cleavedon, BS21 6UW, United \\ Kingdom \\ Darrell.mann@systematic-innovation.com
}

\begin{abstract}
The paper focuses on the identification and resolution of conflicts and contradictions in complex systems. By constructing simple, computerbased bottom-up models of an exemplar complex system, we show the potential for the emergence of multi-phase macro-level outcomes. We then show how these discontinuous phase-shifts may be modelled as contradictions, and from there, how TRIZ tools can be used to generate innovative solutions.
\end{abstract}

Keywords: complexity, emergence, phase-shift, discontinuity, bottom-up

"If you show someone their future, they have no future.

If you take away the mystery, you take away hope."

Philip Dick, Paycheck ${ }^{(1)}$

"Don't get so far ahead of the parade no-one knows you're in it." John Naisbitt ${ }^{(2)}$

\section{Introduction}

What do we mean when we use the word 'innovation'? Opinions vary, but consistent themes include novelty, the addition of value and profit. In this paper we will use a somewhat different definition in order to make an important distinction with 'optimization'. The distinction is especially needed in the computing environment, because while computers are extremely effective at doing the optimization task, they remain to all intents and purposes useless at performing innovation related tasks. Therefore the important distinction between the two things is that 'optimization' involves the identification of optimum balanced values of a known set of continuously variable parameters, whereas 'innovation' involves the presence of some kind of discontinuity. As shown in Figure 1, 'optimization' stays 
within the current paradigm while 'innovation' involves some kind of discontinuous shift (however large or small) to another paradigm. This paper is about the latter. We already know that computers are better than humans at optimization. This is because continuous variables are amenable to mathematical modelling. Discontinuous shifts are generally speaking not.

However, the theme of this paper is that computers already have a profoundly important role to play in helping designers to find those discontinuities. In many technical systems, designers are readily able to find such discontinuities (trade-offs) without the use of a computer. Yet in systems that are fundamentally complex the discontinuities are often hidden from view. This paper examines how in these situations the construction of bottom-up system models and the use of multi-agent, programmable modelling environments can be used not only to identify discontinuities, but to provide designers with a more complete understanding of emergent system level behaviour. It is hoped that in this way, more robust, contradiction-breaking solutions may be identified. Thus we believe that computers already have a significant role to play - one in which they are already far superior to humans - in the innovation arena.

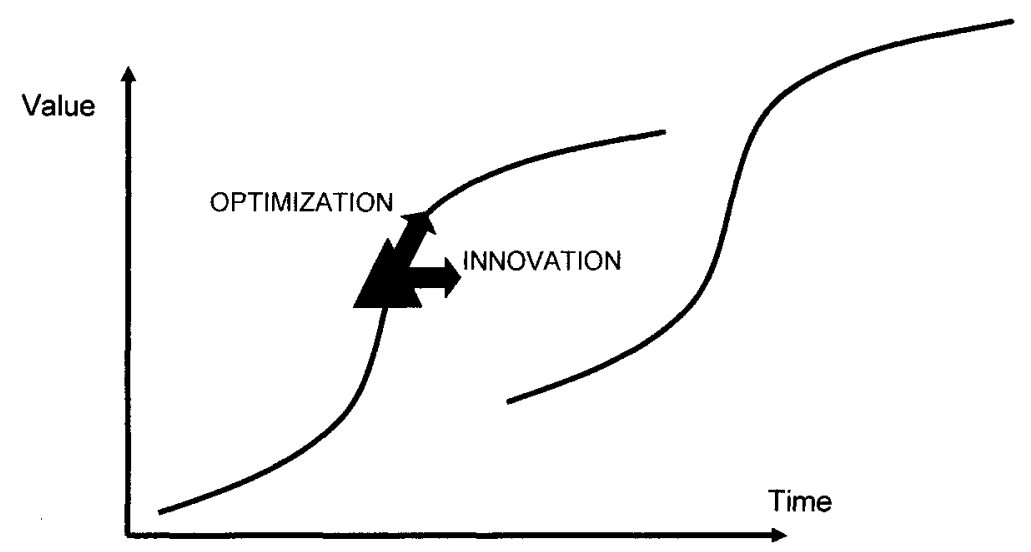

Fig. 1. Fundamental Difference Between 'Optimization' And 'Innovation'

\section{Emergent Complexity - Traffic System Case Study}

The easiest and most effective way to demonstrate the contradiction-finding abilities of computer software is to look at an example. The problem of highway traffic has probably been over-used, but we will examine it anyway. By using TRIZ we might generate one or two new insights into the problem. The traffic problem we will consider is flow and congestion on highways. Several researchers (Reference 3 for example) have sought to model highway traffic flow using multi-agent software models. The main idea here has been to demonstrate that often unexpected complex system-level behaviours will emerge from the combined effects of players (drivers in this case) who can possess no more than local knowledge. 
In Figure 2 we describe some of those 'local' rules as they may apply to an individual driver inside their individual car. In most prior traffic flow study simulations it is assumed that every driver observes these local rules. In our simulations, we have expanded the analysis somewhat by identifying two distinctly different types of driver - one calm and relaxed; the other in a hurry and therefore stressed.

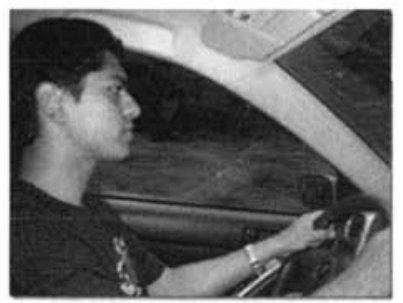

CALM DRIVER

- Drive up to speed limit where possible

- Keep left

- Overtake slow moving vehicle if

sufficient space in outside lane

- Remain safe distance behind vehicle in front

- Drive as fast as possible - Slow to limit if radar/police observed - Overtake slow moving vehicle on any side - Remain small distance behind vehicle in front

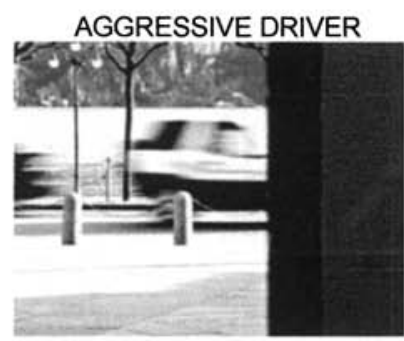

Fig. 2. Defining 'Driver DNA' For Multi-Agent Modeller

While it is clear from other studies that 'all-drivers-follow-the-same-rules' models generate complex behaviour already, one of the aims in this work has been to try and establish the role that driver stress-level might have on emergent traffic behaviour. The simulation models that we built assumed that all drivers defaulted to the 'calm' state. The switch from 'calm' to 'aggressive' behaviour was programmed as a sudden shift in keeping with the 'leaky integrator' model of human brain function (Reference 4). In this model, transitions from one state to another occur abruptly once the level of a chemical messenger reaches a threshold level. Thus, the simulation attempts to model what the world knows about the way the brain operates. When subjected to stressful situations, the body generates more 'stress messengers'. The more stress, the more messengers get produced. At the same time, in order to prevent being drowned in a sea of chemicals, the body gradually disposes of the messengers. Here, then, is the 'leaky' part of the integrator story. An analogy would be filling a container with a small hole in the bottom. If more (stress messenger) fluid is put into the container than is able to drain through the small hole, then the container gradually fills up. If we fill it sufficiently, then eventually the container will become completely full and then start to overflow. This 'over-flow' situation is analogous to the sudden shift from 'calm' to 'aggressive' in the driver model. This is a part of the model that we know mimics reality quite closely. What is less clear is at what point in a highway driving situation drivers will shift from one state to another. In order to model this uncertainty, two stress-generator conditions were incorporated:

1. Stress messengers begin to be generated after drivers have been exposed to stationary traffic for more than a set period of time (this 
period was modelled using a Monte Carlo randomization algorithm in order to reflect the belief that across a population, some people are become stressed more quickly than others); once chemical production has commenced, it will continue each time the driver is stationary after the first occasion.

2. Again controlled by a Monte Carlo algorithm to account for variation across the population, drivers were 'injected' with high rates of stress messengers following any kind of unexpected incident on the road. For example, if another driver braked suddenly or switched lane unexpectedly then this would be modelled as a rapid rise in stress messengers.

It is important to note that during the construction we had little quantified information against which to validate any part of the model. This would be wholly unacceptable if our task was to try and 'optimize' any part of the highway traffic flow problem, but the approach is valid for identifying non-linearities. While incorrect quantifications might result in us getting things in the wrong position if we tried to plot results on an absolute numerical grid, we are able to identify and model the relative situations and non-linear phenomena.

Once the rules were defined we ran multiple multi-agent software (Reference 5) simulations. Of primary interest during these simulations was the speed vehicles were capable of travelling during different traffic density conditions. Figure 3 shows a typical output when the simulations are used to plot the distance travelled along the highway by a succession of vehicles plotted as a function of time. What we should be looking for ideally in these plots is a succession of lines with constant gradients as in the left hand picture (gradient here being representative of speed).

Without going in to the specific details of what is shown in the right-hand Figure, what we actually see is very different. This is a plot describing the motion of many cars. The sharp ridges in the plots are indicative of sudden changes in speed of the vehicles now that the overall traffic density has exceeded a certain threshold value.

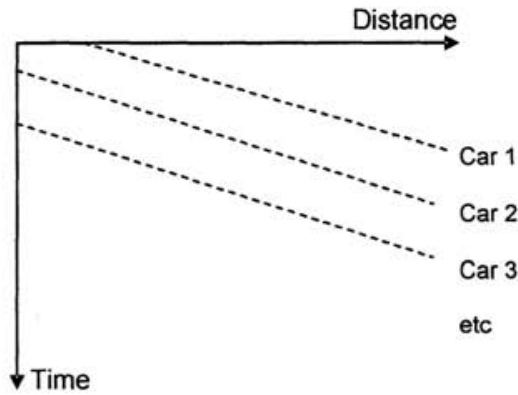

'Free Flow'

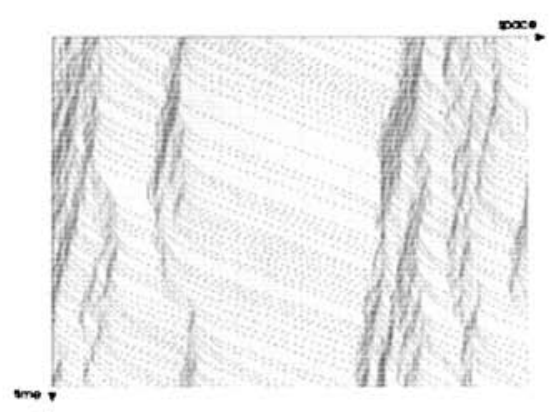

'Pulse' Flow

Fig. 3. Emerging Discontinuous Phase Transitions

In other words drivers all following the same set of linear rules can create a system behavior that is highly non-linear. If we analyze all of these non-linearities in 
detail, what we discover is that there are a number of very distinct modes of highway traffic flow. We can see these different modes plotted in Figure 4.

The reader may recognize some of these modes from their own personal highway driving experiences:

1. 'free-flow' traffic - in this mode, an individual driver is able to proceed unhindered by the effects of other traffic.

2. 'knotted-flow' - once the traffic density passes a certain value, the occasional slow vehicle on the highway tends to impede the progress of other vehicles. These 'knots' mean that other drivers are temporarily impeded in their progress until such times as they are able to access overtaking lanes and get past the slow moving impedance.

3. 'pulse' flow - this is perhaps the strangest phase of the four. The traffic speed oscillates, often wildly, between low and normal speeds; one second vehicles are driving normally, the next everyone is braking, then a few seconds later, vehicles rapidly accelerate up to normal speed again.

4. 'choked' flow - the most frustrating mode. Drivers find themselves stationary for periods, followed by periods of crawling progress.

The important thing to note here is that these four modes are discontinuously different from one another, undergoing a rapid transition from one mode to another. A useful analogy to keep in mind is the discontinuous phase transition that occurs when water is chilled to below zero degrees; one second it is liquid, the next ice. The transition from one mode to another occurs suddenly and with little or often no warning. The transitions offer the first seeds of opportunity for defining good contradictions to solve. However, it is worth first exploring in a little more detail how and why these phase transitions occur.

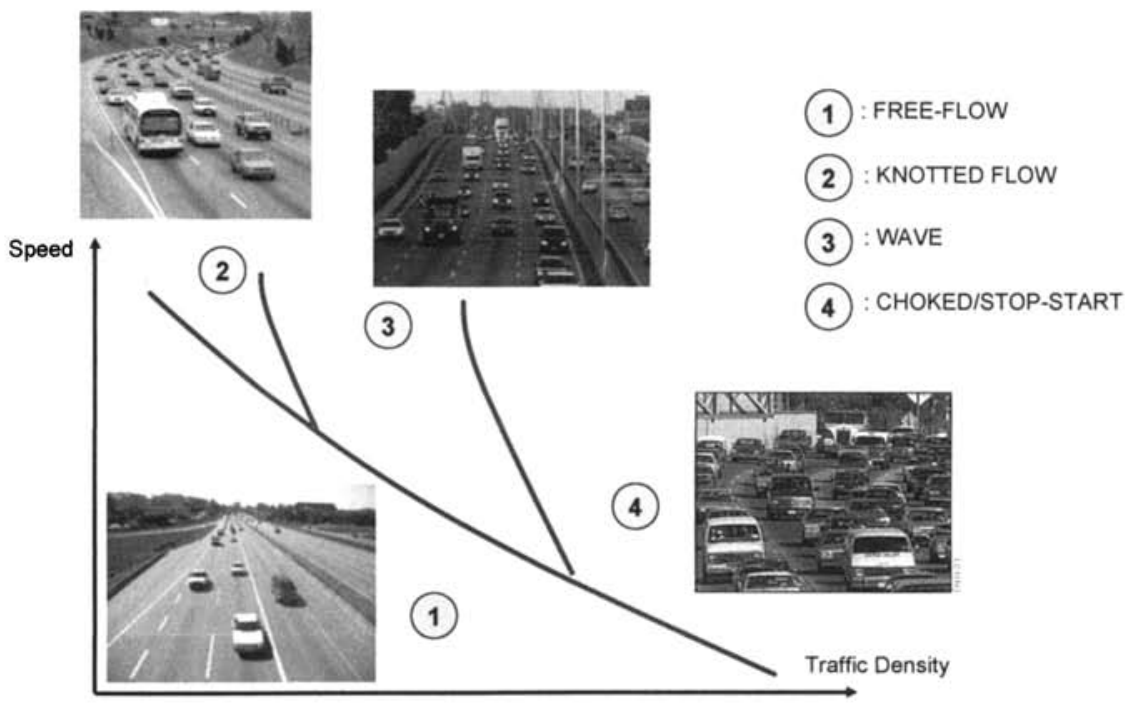

Fig. 4. Phase Transitions In Highway Traffic Flow 
Emphasizing again the crucial distinction between optimization and innovation, the values of temperature, pressure, etc at which water makes the abrupt transition to ice are accurately known because thousands of experiments have been conducted. Yet even if we didn't know the precise freezing temperature of water, it would still be possible to clearly see the non-linear transition from liquid to solid taking place. In the case of the traffic simulation presented here, no experimental validation has taken place and so there is no possibility to state the actual speeds and traffic densities that denote the transition from one mode to another. Hence there has been no attempt to include numbers on the axes of the graph. Our interest rests with innovation, and the working hypothesis is that here the job is first and foremost about identifying the existence of non-linear transitions. Once the innovative solution concepts have been developed following the identification of the non-linear problems, we can worry about quantification and optimization.

\subsection{Sub- And Super-Critical Phenomena}

In the same way that it is possible to cool water below its zero degree 'critical point' and for it still to be a liquid, it is eminently possible for traffic to be travelling in one phase mode even though the speed-versus-density conditions indicate that it should be in another. In physics this condition is called 'super-critical'. Given the right conditions, water, can be cooled several degrees below zero and it still will not freeze. As soon as this 'super-critical' condition experiences a sudden perturbation (for example the presence of a tiny solid particle in the water acts as an ice formation initiation point), the whole system will rapidly switch to ice.

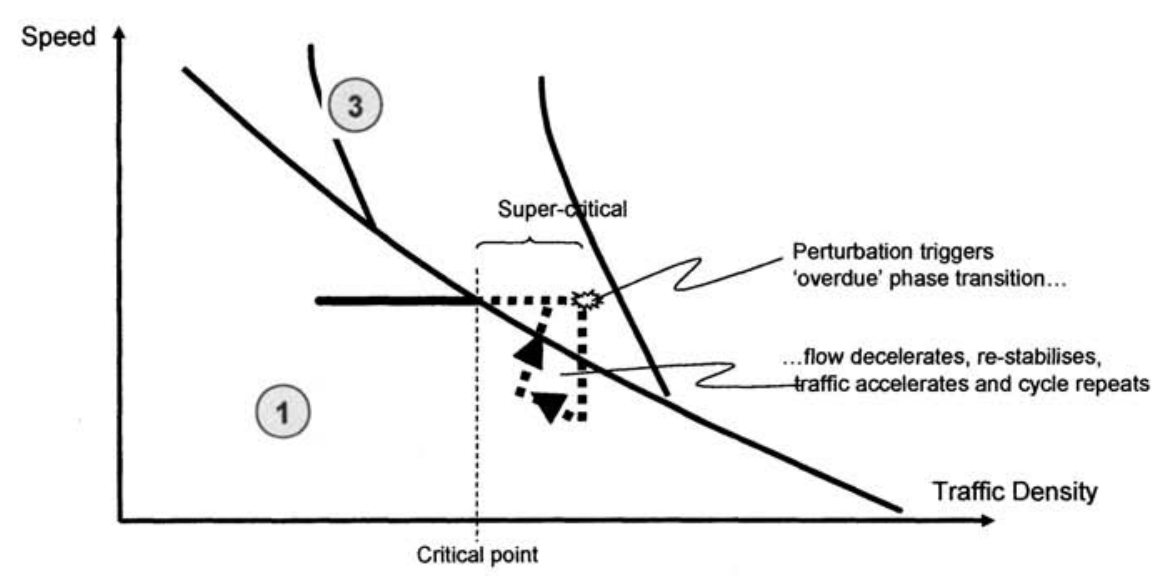

Fig. 5. Super-Critical Behaviour In Highway Traffic Flow And Influence Of Perturbations

The same thing occurs with traffic flow when any phase boundary is crossed. Figure 5 illustrates one such example. It is perfectly possible for the traffic flow to transition over a phase boundary without the transition occurring. However, like the 
initiation point in ice formation, as soon as an appropriate perturbation occurs in the traffic (a driver suddenly changes lane, new vehicles enter at an on-ramp, lanes narrow at road-works, etc) then the phase transition will occur. As shown in Figure 5 , taking the transition between free-flow and wave-flow as its example, the perturbation causes the vehicle speed to drop suddenly. Once it drops sufficiently the phase boundary is crossed again and within a short time vehicles are able to accelerate, thus decreasing density. However the density decrease is temporary and so we get back to where we started in the cycle; flow at the original speed and density until another perturbation occurs.

\subsection{Phase Transitions Define Contradictions}

Returning to the idea that phase boundaries define contradictions, the connection we make here involves the idea that phase boundaries and contradictions both involve the concept of discontinuity. Just as the discontinuous shift between water and ice means presents a 'solid and liquid' contradiction, the same thing happens across the boundary between two traffic phases. Free-flow traffic behaviour is fundamentally different from pulse-flow behaviour. Just as ice and water can't be treated in the same way, we ought not to treat different traffic phases in the same way. In many ways, when TRIZ recommends the use of Principle 35, Parameter Changes it is a prompt for us to look not for optimizing parameter shifts, but rather shifts that transition across some kind of discontinuous boundary (Ref 6).

The idea that different phases act on different sides of a contradiction, is intended to give a clear indication that there is no such thing as an 'optimum' solution to this type of multi-phase problem. Thus any attempt to manage traffic using a single solution (by for example imposing a speed limit) is destined to work unsatisfactorily on both sides of the phase shift boundary. This phenomenon then connects us to another important idea, this time one from the world of cybernetics:

\section{Law Of Requisite Variety}

Cybernetics pioneer W Ross Ashby stated (Reference 7) 'only variety can absorb variety' - Figure 6 . This apparently obvious and over-simple statement hides a mass of important ideas. As far as our traffic control problem is concerned, as well as reenforcing the contradiction idea that there can be no single solution to a situation. bearing multiple discontinuous phases, it informs tells us that there needs to be a level of variety in the potential solution that matches the variety present in the system we are trying to control. This means that the variety in the four-phases should by rights require different treatment. 


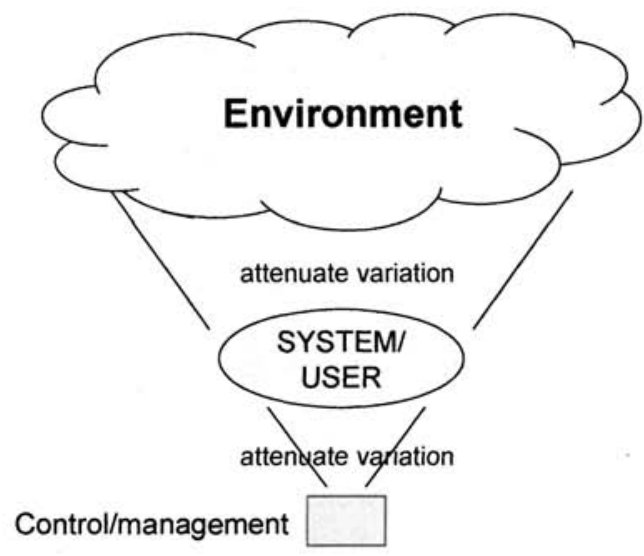

Fig. 6. Only Variety Can Absorb Variety

\section{Solving The Contradictions}

In geographical locations where the traffic problem has become bad enough, we can see how road planners have made at least one attempt to manage two of our four traffic phases. The basic contradiction for all four of the phases is one between speed and density (or 'speed' versus 'amount of substance' in TRIZ Contradiction Matrix terms). Figure 7 illustrates how this conflict pair has been mapped onto the latest version of the Matrix (Reference 7), and then how one of the suggested Inventive Principles in turn can be translated into the variable speed limit system now found in some busy road systems.

This 'variable speed limit' solution is an attempt to recognise the first mode ('free-flow') versus other modes (2, 3 and 4 in Figure 4) contradiction. The traffic control system in these solutions measures traffic density and sets the speed limit accordingly.

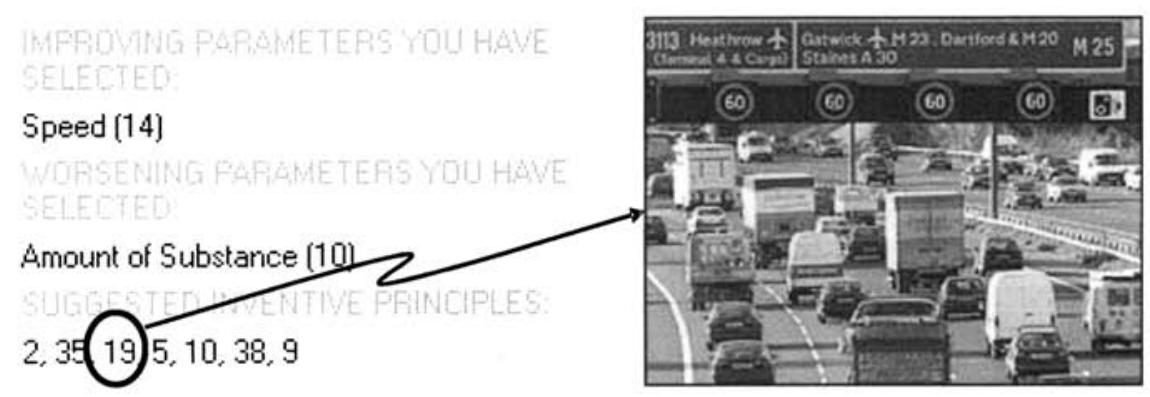

Fig. 7. Variable speed limit control systems work for one phase but not necessarily others 
Variable speed limit systems represent a partial answer, but not (as the designers probably intended) the answer to the highway traffic congestion problem. The phase diagram and the Law of Requisite variety inform us why this is the case: Four discontinuously different phases require four different solutions. We experience the compromise presented by the current systems when we find ourselves sitting in a stationary car (i.e. mode 4) being told that the speed limit is $50 \mathrm{mph}$. Choked/stopstart mode traffic requires a different solution than mode 3, 'pulsed flow' - where the variable speed limit solution actually works.

Knowing that four phases require four discontinuously different solutions causes us to return to the TRIZ Contradiction tools in order to identify further contradiction breaking solutions.

Staying with the contradiction parameters and Inventive Principle recommendations shown in Figure 7, the following conceptual solutions may help designers to better manage the four distinct traffic modes:

- Principle 5 (Merging) - combine the information generated at multiple road sensors in order to detect the presence or otherwise of 'pulsing' traffic behaviour. If no pulsing, then switch to an algorithm appropriate to other modes - e.g. in Mode 2 traffic, it may be advisable to display no message on the traffic signs.

- Principle 35 (Parameter Change) - in Mode 4 traffic it may be better to display another symbol other than a speed limit. For example a 'danger' or 'accident hazard' icon might be a better way to warn drivers that the traffic is in a dangerous state. A possible strategy here might be to display a delay time rather than a speed limit - playing on the well-known psychological phenomenon that people are far more likely to become stressed when they don't know what is happening and how long a problem will last than they would if they knew when the problem was likely to go away. Even if the news is bad (i.e. displaying a '15 minute delay' indication) it is preferable to no news because knowing the extent of the delay in this case would allow drivers to contemplate turning off their engine and reading a book.

- Principle 9 (Prior Counter-Action) - this suggests the idea of 'pre-tensioning' the traffic flow - perhaps by allowing a higher speed limit (i.e. above national limit) immediately after a blockage in order to unchoke the flow at the bottleneck. Or perhaps it might mean pre-warning drivers of emerging problems at longer distances further along the road - i.e. give drivers a message before they see the reduced speed limit signs.

The main point to note at this stage is that in this situation, only a computer simulation has been able to identify the full extent of the traffic problem. As far as traffic control designers are concerned, they have generated a partial solution to the problem (in all likelihood unconscious that they have solved a contradiction!), but partial is all it is. Computer aided innovation in this sense - and we think it generalizes to all complex, multi-phase problems - is about finding the contradictions that would otherwise be hidden. At the moment, the designer then needs to do the creative - solution generation - part of the innovation. But then this is the part most designers enjoy the most.

Later on, the optimizers can do their real world experiments and, with the help of the computers again (computers being excellent optimizers), design the actual quantified traffic control algorithms. This is, of course, a critical part of the solution 
implementation story, albeit it is one that has little if anything to do with the job of innovating.

\section{Final Thought}

The automotive industry already knows they can design automated vehicle systems - e.g. braking - that offer considerable safety benefits relative to even the best driver. And yet they also understand human psychology well enough to know that if control of critical activities like steering, accelerating or braking is taken away from the driver, the driver will not purchase the vehicle. People like to feel like they are in control of their own destiny. As suggested by the Philip Dick quotation at the beginning of this paper, CAI has a similar dilemma to the automotive designer. Take away control from the human designer by creating a 'computerised innovator' and, if the automotive analogy holds true, no-one will use it. On the other hand, present it in such a way that it offers new opportunities to employ their creative skills - as we think is the case with multi-agent programmable modelling environments - and there is a strong likelihood of a win-win situation; the computers do what they're good at (in this case finding what the human alone cannot); the human then gets to dream the breakthrough solution.

\section{References}

1. Dick, P., 'Paycheck', Gollancz, 2004.

2. Naisbitt, J., 'Mind Set!: Reset Your Thinking and See the Future', HarperCollins, 2006.

3. Resnick, M., 'Turtles, Termites and Traffic Jams: Explorations in Massively Parallel Microworlds (Complex Adaptive Systems)', MIT Press, New Edition, 1997.

4. Grand, S., 'Creation: Life And How To Make It', Weidenfeld \& Nicolson, 2000.

5. Wilensky, U., 'NetLogo', http://ccl.northwestern.edu/netlogo/. Center for Connected Learning and Computer-Based Modeling, Northwestern University, Evanston, IL, 1999.

6. Systematic Innovation E-Zine, 'Effective Use Of Principle 35', Issue 58, January 2007.

7. Ashby, W.R., 'An Introduction to Cybernetics', Chapman \& Hall, London, 1957, available electronically at http://pespmc1.vub.ac.be/books/IntroCyb.pdf.

8. Matrix+ Software, www.systematic-innovation.com, 2007. 